Real Analysis Exchange

Vol. 23(2), 1997-1998, pp. 743-752

Ewa Strońska, Mathematics Department, Pedagogical University, ul.

Chodkiewicza 30, 85-064 Bydgoszcz, Poland. e-mail

strewa@wsp. bydgoszcz.pl

\title{
ON THE MAXIMAL FAMILIES FOR SOME SPECIAL CLASSES OF STRONGLY QUASI-CONTINUOUS FUNCTIONS
}

\begin{abstract}
The maximal families (additive, multiplicative, lattice and with respect to the composition) for some special classes of strongly quasicontinuous functions are investigated.
\end{abstract}

Let $\mathcal{R}$ be the set of all reals and let $\mu_{e}(\mu)$ denote the outer Lebesgue measure (the Lebesgue measure) in $\mathcal{R}$. Denote by

$$
\begin{gathered}
d_{u}(A, x)=\limsup _{h \rightarrow 0^{+}} \mu_{e}(A \cap(x-h, x+h)) / 2 h \\
\left(d_{l}(A, x)=\liminf _{h \rightarrow 0^{+}} \mu_{e}(A \cap(x-h, x+h)) / 2 h\right.
\end{gathered}
$$

the upper (lower) density of a set $A \subset \mathcal{R}$ at a point $x$. A point $x \in \mathcal{R}$ is called a density point of a set $A \subset \mathcal{R}$ if there exists a measurable (in the sense of Lebesgue) set $B \subset A$ such that $d_{l}(B, x)=1$. The family

$\mathcal{T}_{d}=\{A \subset \mathcal{R} ; A$ is measurable and every point $x \in A$ is a density point of $A\}$ is a topology called the density topology [1]. Denote by $\operatorname{int}(A)$ the interior (Euclidean) of the set $A$. The family

$$
\mathcal{T}_{\text {ae }}=\left\{A \in \mathcal{T}_{d} ; \mu(A-\operatorname{int}(A))=0\right\}
$$

is also a topology [5].

A function $f$ (from $\mathcal{R}$ into $\mathcal{R}$ ) is called $\mathcal{T}_{\text {ae }}-$ continuous $\left(\mathcal{T}_{d}-\right.$ continuous or approximately continuous) at a point $x$ if it is continuous at $x$ as the application from $\left(\mathcal{R}, \mathcal{T}_{a e}\right)$ (from $\left(\mathcal{R}, \mathcal{T}_{d}\right)$ ) into $\left(\mathcal{R}, \mathcal{T}_{e}\right)$, where $\mathcal{T}_{e}$ denotes the

Key Words: continuity, strong quasicontinuity, density topology, maximal additive and multiplicative families

Mathematical Reviews subject classification: 26A15, 54C08, 54C30

Received by the editors April 4, 1997

*Supported by Bydgoszcz WSP grant (1996) 
Euclidean topology in $\mathcal{R}$. A function $f$ is $\mathcal{T}_{a e}-$ continuous (everywhere on $\mathcal{R}$ ) if and only if it is $\mathcal{T}_{d}$ - continuous (everywhere) and almost everywhere (relative to $\mu$ ) continuous [5]. A function $f$ is said to be strongly quasicontinuous (in short s.q.c.) at a point $x$ if for every set $A \in \mathcal{T}_{d}$ containing $x$ and for every positive real $\eta$ there is an open interval $I$ such that $I \cap A \neq \emptyset$ and $|f(t)-f(x)|<\eta$ for all $t \in A \cap I$ [2]. If a function $f$ is s.q.c-continuous at every point then we say that $f$ is s.q.c-continuous.

In this paper the main results are some modifications of the results of Z.Grande in [4].

Let $\mathcal{P}(x)$ be a property of a function $f$ at a point $x$ (we will write $f \in \mathcal{P}(x)$ ) such that:

if $f$ is continuous at $x$ then $f \in \mathcal{P}(x)$;

if $f \in \mathcal{P}(x)$ then $-f \in \mathcal{P}(x)$;

if $f \in \mathcal{P}(x)$ and $g / I=f / I$ for some open interval $I$ containing $x$ then $g \in \mathcal{P}(x)$.

Denote by $P$ the family of all functions $f$ such that for every positive real $\eta$ and for every point $x$ and for every set $A \in \mathcal{T}_{d}$ containing $x$ there is an open interval $I$ such that $I \cap A \neq \emptyset,|f(t)-f(x)|<\eta$ and $f \in \mathcal{P}(t)$ for all $t \in I \cap A$.

Now, let:

$-C=\{f ; f$ is continuous $\}$;

- $C_{a e}=\left\{f ; f\right.$ is $\mathcal{T}_{a e}-$ continuous $\}$;

- $Q_{s}=\{f ; f$ is s.q.c. $\}$;

- $\operatorname{Max}_{\text {add }}(P)=\{f ; f+g \in P$ for every $g \in P\}$;

- $\operatorname{Max}_{\text {mult }}(P)=\{f ; f g \in P$; for every $g \in P\}$;

- $\operatorname{Max}_{\max }(P)=\{f ; \max (f, g) \in P$ for every $g \in P\}$;

- $\operatorname{Max}_{\min }(P)=\{f ; \min (f, g) \in P$ for every $g \in P\}$;

- $\operatorname{Max}_{\text {comp }}(P)=\{f ; f \circ g \in P$ for every $g \in P\}$.

Remark 1. Evidently

$$
C \subset P \cup C_{a e} \subset Q_{s} .
$$

So, every function $f \in P$ is almost everywhere continuous [2, 3]. 
Remark 2. The inclusion

$$
\operatorname{Max}_{a d d}(P) \cup \operatorname{Max}_{\text {mult }}(P) \cup \operatorname{Max}_{\max }(P) \cup \operatorname{Max}_{\min }(P) \cup \operatorname{Max}_{\text {comp }}(P) \subset P
$$

is true.

Proof. Since the functions $g_{1}(t)=0, g_{2}(t)=1$ and $g_{3}(t)=t$ for $t \in$ $\mathcal{R}$ belong to $P$, for all functions $f_{1} \in \operatorname{Max}_{\text {add }}(P), f_{2} \in \operatorname{Max}_{\text {mult }}(P)$ and $f_{3} \in \operatorname{Max}_{\operatorname{comp}}(P)$ we obtain have $f_{1}=f_{1}+g_{1} \in P, f_{2}=f_{2} g_{2} \in P$ and $f_{3}=f_{3} \circ g_{3} \in P$. So,

$$
\operatorname{Max}_{\text {add }}(P) \cup \operatorname{Max}_{\text {mult }}(P) \cup \operatorname{Max}_{\text {comp }}(P) \subset P .
$$

If a function $f$ is not in $P$ then there are a positive real $\eta$, a point $x$ and a set $A \in \mathcal{T}_{d}$ containing $x$ such for every open interval $I$ with $I \cap A \neq \emptyset$ there is a point $t \in I \cap A$ such that $|f(t)-f(x)| \geq \eta$ or $f$ is not in $\mathcal{P}(t)$. Then the functions $\max (f, f(x)-\eta)$ and $\min (f, f(x)+\eta)$ are not in $P$. So, $f$ is not in $\operatorname{Max}_{\max }(P) \cup \operatorname{Max}_{\min }(P)$ and the proof is completed.

I. The family $\operatorname{Max}_{a d d}(P)$.

In this part we suppose that the property $\mathcal{P}(x)$ is such that if $f, g \in \mathcal{P}(x)$ then $f+g \in \mathcal{P}(x)$ (then we say that $\mathcal{P}()$ has the additive property).

Theorem 1. Assume $\mathcal{P}(x)$ has the additive property. Then

$$
C_{a e} \cap P=\operatorname{Max}_{a d d}(P)
$$

holds.

Proof. Let $f \in C_{a e} \cap P$ and $g \in P$ be functions. Fix a positive real $\eta$, a point $x$ and a set $A \in \mathcal{T}_{d}$ containing $x$. Since $f \in C_{a e}$, the point $x$ is a density point of the set $B=\operatorname{int}(\{t ;|f(t)-f(x)|<\eta / 2\})$. Consequently, $x$ is a density point of the set $B \cap A$. Since $g \in P$, there is an open interval $J \subset B$ such that $J \cap A \neq \emptyset,|g(t)-g(x)|<\eta / 2$ and $g \in \mathcal{P}(t)$ for every $t \in J \cap A$. From the relation $f \in P$ follows that there is an open interval $I \subset J$ such that $I \cap A \neq \emptyset$ and $f \in \mathcal{P}(t)$ for all points $t \in I \cap A$. Consequently, $I \cap A \neq \emptyset, f+g \in \mathcal{P}(t)$ and $|(f(t)+g(t))-(f(x)+g(x))|<\eta / 2+\eta / 2=\eta$ for all points $t \in I \cap A$. So, the function $f \in \operatorname{Max}_{a d d}(P)$ and the inclusion $C_{a e} \cap P \subset \operatorname{Max}_{a d d}(P)$ is proved.

For the proof of the inclusion $\operatorname{Max}_{a d d}(P) \subset C_{a e} \cap P$ fix a function $f \in$ $\operatorname{Max}_{a d d}(P)$. By Remark 1 the function $f \in P$. If $f$ is not in $C_{a e}$ then there are a point $x \in \mathcal{R}$ and a positive number $\eta$ such that the closure $c l(\{t ; \mid f(t)-$ $f(x) \mid>\eta\})$ of the set $\{t ;|f(t)-f(x)|>\eta\}$ has positive upper density at a point $x$. We can assume that the closure

$$
c l(\{t ; f(t)>f(x)+\eta\})
$$


has positive upper density at a point $x$. Since $f$ belonging to $P \subset Q_{s}$ is almost everywhere continuous $[2,3]$, we obtain

$$
\mu(c l(\{t ; f(t)>f(x)+\eta\}) \backslash\{t ; f(t) \geq f(x)+\eta\})=0
$$

and consequently,

$$
d_{u}(\operatorname{int}(\{t ; f(t)>f(x)+\eta / 2\}), x)>0 .
$$

Thus there is a sequence of disjoint closed intervals $I_{n}=\left[a_{n}, b_{n}\right] \subset\{t ; f(t)>$ $f(x)+\eta / 2\}, n=1,2, \ldots$, such that:

(1) $x$ is not in $I_{n}$ for $n=1,2, \ldots$;

(2) $f$ is continuous at all points $a_{n}, b_{n}, n=1,2, \ldots$;

(3) $\lim _{n \rightarrow \infty} a_{n}=\lim _{n \rightarrow \infty} b_{n}=x$;

(4) $d_{u}\left(\bigcup_{n} I_{n}, x\right)>0$.

Put

$$
g(t)=\left\{\begin{array}{cc}
-f(x)+\eta / 2 & \text { if } \\
-f(t) & \text { otherwise. }
\end{array} \quad(t=x) \vee\left(t \in I_{n}, n=1,2, \ldots\right)\right.
$$

Fix a positive real $\eta$, a point $t$ and a set $A \in \mathcal{T}_{d}$ containing $t$. For a positive integer $n$ and a point $t=a_{n}$ or $t=b_{n}$ the function $g$ is unilaterally continuous at $t$ and $g / I_{n}$ is constant. So, there is an open interval $I \subset I_{n}$ with $I \cap A \neq \emptyset$. Evidently, $g \in \mathcal{P}(u)$ and $|g(u)-g(x)|=0<\eta$ for each point $u \in I \cap A$. If $t \in \operatorname{int}\left(I_{n}\right)$ for some positive integer $n$ we proceed the same as above. If $t \neq x$ and $t$ is not in $I_{n}$ for $n=1,2, \ldots$ then there is an open interval $I$ with $I \cap I_{n} \neq \emptyset$ for $n=1,2, \ldots, I \cap A \neq \emptyset$ and such that $\mid f(u)-f(t \mid<\eta$ and $f \in \mathcal{P}(u)$ for $u \in I \cap A$. Since $g / I=-f / I$, we obtain $|g(u)-g(t)|=|f(u)-f(t)|<\eta$ and $g \in \mathcal{P}(u)$ for all points $u \in I \cap A$. If $t=x$ then, by (4), there is a positive integer $n$ with $A \cap \operatorname{int}\left(I_{n}\right) \neq \emptyset$. Since $g(u)=-f(x)+\eta / 2$ for $u=x$ and for $u \in \operatorname{int}\left(I_{n}\right)$, we have $g \in \mathcal{P}(u)$ and $|g(u)-g(t)|=0<\eta$ for $u \in A \cap \int\left(I_{n}\right)$. So, $g \in P$. Moreover, $f(x)+g(x)=\eta / 2, f(t)+g(t) \geq \eta$ for $t \in I_{n}, n=1,2, \ldots$ and $f(t)+g(t)=0$ otherwise on $\mathcal{R}$. So, $f+g$ is not in $P$ and consequently $f$ is not in $\operatorname{Max}_{a d d}(P)$. This contradiction finishes the proof.

II.The families $\operatorname{Max}_{\max }(P)$ and $\operatorname{Max}_{\min }(P)$.

In this part we suppose about the property $\mathcal{P}(x)$ that if $f, g \in \mathcal{P}(x)$ then also $\max (f, g), \min (f, g) \in \mathcal{P}(x)$ (then we say that $\mathcal{P}()$ has the lattice property). 
Theorem 2. Let $\mathcal{P}(x)$ has the lattice property. Then

$$
\operatorname{Max}_{\max }(P)=\operatorname{Max}_{\min }(P)=C_{a e} \cap P
$$

holds.

Proof. For the proof of the inclusion

$$
C_{a e} \cap P \subset \operatorname{Max}_{\max }(P) \cap \operatorname{Max}_{\min }(P) .
$$

we take a function $f \in C_{a e} \cap P$ and a function $g \in P$. Fix a positive real $\eta$, a point $x$ and a set $A \in \mathcal{T}_{d}$ containing $x$. Let $h=\max (f, g)$. Consider the following cases:

(1) $f(x)>g(x)$. Then let $r=f(x)-g(x)$ and let $s=\min (r / 2, \eta)$. Since $f \in C_{a e}, x$ is a density point of the set $B=\operatorname{int}(\{t ;|f(t)-f(x)|<s\})$. From the relation $g \in P$ follows that there is an open interval $J \subset B$ such that $J \cap A \neq \emptyset, g \in \mathcal{P}(t)$ and $|g(t)-g(x)|<s$ for all points $t \in J \cap A$. Since $f \in P$, there is an open interval $I \subset J$ with $I \cap A \neq \emptyset$ and $f \in \mathcal{P}(t)$ for all points $t \in I \cap A$. Observe that for $u \in I \cap A$ we have

$$
f(u)>f(x)-s \geq g(x)+2 s-s=g(x)+s>g(u),
$$

whence $h(u)=f(u)$. Moreover, $h(x)=f(x), h \in \mathcal{P}(u)$ and

$$
|h(u)-h(x)|=|f(u)-f(x)|<s \leq \eta
$$

for all point $u \in I \cap A$.

(2) $f(x)<g(x)$. In this case the proof is analogous as above.

(3) $f(x)=g(x)$. In this case we put $s=\eta$ and we find an open interval as above. Then $I \cap A \neq \emptyset$ and for $u \in I \cap A$ we obtain $h \in \mathcal{P}(u)$ and

$$
|h(u)-h(x)| \leq \max (|f(u)-f(x)|,|g(u)-g(x)|)<s=\eta .
$$

So, $h=\max (f, g) \in P$. The prof that $\min (f, g) \in P$ is analogous.

Since by Remark 1 the inclusion $\operatorname{Max}_{\max }(P) \cup \operatorname{Max} x_{\min }(P) \subset P$ is true, we will show the inclusion $\operatorname{Max}_{\max }(P) \cup \operatorname{Max}_{\min }(P) \subset C_{a e}$. We will show only that $\operatorname{Max}_{\max }(P) \subset C_{a e}$, because the proof of the inclusion $\operatorname{Max}_{\min }(P) \subset C_{a e}$ is similar. Let $f \in \operatorname{Max}_{\max }(P)$ be a function. By Remark 1 the function $f \in P$. If $f$ is not in $C_{a e}$ then there are a point $x$ and a positive number $\eta$ such that

$$
d_{u}(\operatorname{cl}(\{t ;|f(t)-f(x)|>\eta\}), x)>0 .
$$

If

$$
d_{u}(c l(\{t ; f(t)>f(x)+\eta\}), x)>0
$$


then the same as in the proof of Theorem 1 there are disjoint closed intervals

$$
I_{n}=\left[a_{n}, b_{n}\right] \subset\{t ; f(t)>f(x)+\eta / 2\},
$$

such that conditions (1) - (4) from the proof of Theorem 1 are satisfied. Let

$$
g(t)=\left\{\begin{array}{cc}
f(x)-\eta & \text { if } \\
f(x)+\eta & \text { otherwise. }
\end{array} \quad(t=x) \vee\left(t \in I_{n}, n=1,2, \ldots,\right)\right.
$$

Analogously as in the proof of Theorem 1 we can show that $g \in P$. Moreover, $\max (f(x), g(x))=f(x)$ and $\max (f(t), g(t)) \geq f(x)+\eta / 2$ for $t \neq x$. So, $\max (f, g)$ is not in $P$ and consequently, $f$ is not in $\operatorname{Max}_{\max }(P)$. Now consider the case where

$$
d_{u}(c l(\{t ; f(t)<f(x)-\eta\}), x)>0
$$

- Then there are disjoint closed intervals $I_{n}=\left[a_{n}, b_{n}\right] \subset\{t ; f(t)<f(x)-$ $\eta / 2\}, n=1,2, \ldots$, which satisfy conditions (1)-(4) from the proof of Theorem 1. Let the function $g$ be defined the same as above. Then $g \in P$, $\max (f(x), g(x))=f(x), \max (f(t), g(t)) \leq f(x)-\eta / 2$ for $t \in I_{n}, n=1,2, \ldots$, and $\max (f(t), g(t)) \geq f(x)+\eta$ otherwise on $\mathcal{R}$. So,in this case also $\max (f, g)$ is not in $P$, and consequently $f$ is not in $\operatorname{Max}_{\max }(P)$. This contradiction finishes the proof.

III. The family $\operatorname{Max}_{c o m p}(P)$.

In this part we suppose that for every continuous function $g$ and for every function $f \in \mathcal{P}(x)$ we have $g \circ f \in \mathcal{P}(x) ; \mathcal{P}()$ is invariant with respect to composition with continuous function.

Theorem 3. Assume $\mathcal{P}(x)$ is invariant with respect to composition with continuous function. Then

$$
\operatorname{Max}_{c o m p}(P)=C
$$

holds.

Proof. Let $g$ be a continuous function and let $f \in P$ be a function. Fix a positive real $\eta$, a point $x$ and a set $A \in \mathcal{T}_{d}$ containing $x$. Since $g$ is continuous at $f(x)$, there is a positive real $r$ such that if $|u-f(x)|<r$ then $|g(u)-g(f(x))|<$ $\eta$. From the relation $f \in P$ follows that there is an open interval $I$ such that $I \cap A \neq \emptyset, f \in \mathcal{P}(t)$ and $|f(t)-f(x)|<r$ for all points $t \in I \cap A$. Observe that for every point $t \in I \cap A$ we obtain $g \circ f \in \mathcal{P}(t)$ and $|g(f(t))-g(f(x))|<\eta$. So, $g \circ f \in P$ and consequently $C \subset \operatorname{Max}_{\text {comp }}(P)$.

Suppose that a function $f$ is not continuous at a point $y$. Then there is a sequence of points $y_{n} \neq y, n=1,2, \ldots$, such that $\lim _{n \rightarrow \infty} y_{n}=y$ and $\lim _{n \rightarrow \infty} f\left(y_{n}\right) \neq f(y)$. Let $I_{n}=\left[a_{n}, b_{n}\right], n=1,2, \ldots$, be disjoint closed intervals such that 


$$
\begin{aligned}
& -\lim _{n \rightarrow \infty} a_{n}=\lim _{n \rightarrow \infty} b_{n}=0 ; \\
& -a_{n} b_{n}>0 \text { for } n=1,2, \ldots, ; \\
& -d_{u}\left(\bigcup_{n} I_{n}, 0\right)>0 .
\end{aligned}
$$

Put

$$
g(x)=\left\{\begin{array}{ccl}
y_{n} & \text { if } & x \in I_{n}, n=1,2, \ldots, \\
y & \text { if } & x=0 \\
y_{1} & \text { otherwise. } &
\end{array}\right.
$$

Fix a positive real $\eta$, a point $x$ and a set $A \in \mathcal{T}_{d}$ containing $x$. If $x \neq 0$ then $g$ is unilaterally continuous and consequently, there is an open interval $I$ such that $I \cap A \neq \emptyset, g$ is continuous at every point $t \in I$ and $g(t)=g(x)$ for each point $t \in I$. If $x=0$ then there is a positive integer $n$ such that $\left|y_{n}-y\right|<\eta$ and $I_{n} \cap A \neq \emptyset$. Consequently, there is an open interval $I \subset I_{n}$ with $I \cap A \neq \emptyset$. Observe that the reduced function $g / I$ is continuous and $|g(u)-g(x)|=\left|y_{n}-y\right|<\eta$ for $u \in I$. This shows that $g \in P$. But $f \circ g$ is not in $P$, since $f \circ g$ is not s.q.c. at $x=0$. So, $\operatorname{Max}_{\text {comp }}(P) \subset C$, and the proof is completed.

IV. The family $\operatorname{Max}_{\text {mult }}(P)$.

In this part we suppose about the property $\mathcal{P}(x)$ that:

- if $f, g \in \mathcal{P}(x)$ then $f g \in \mathcal{P}(x)$;

- if $f \in \mathcal{P}(x)$ and $I$ is an open interval such that 0 is not in $f(I)$ then the function

$$
g(t)=\left\{\begin{array}{cc}
1 / f(t) & \text { for } \\
0 & \text { otherwise. }
\end{array} \quad t \in I\right.
$$

belongs to $P$.

Remark 3. If a function $f \in P$ is not $\mathcal{T}_{a e}-$ continuous at a point $x \in \mathcal{R}$ at which $f(x) \neq 0$ then there is a function $g \in P$ such that the product $f g$ is not in $P$.

Proof. The same as in the proof of Theorem 1 we prove that there exist a positive real $\eta$ and disjoint closed intervals $I_{n}=\left[a_{n}, b_{n}\right] \subset\{t ;|f(t)-f(x)|>$ $\eta / 2\}$ which satisfy conditions (1)-(4) from the proof of Theorem 1. Put

$$
g(t)=\left\{\begin{array}{ccc}
1 & \text { if } & (t=x) \vee\left(t \in I_{n}, n=1,2, \ldots,\right) \\
0 & \text { otherwise. }
\end{array}\right.
$$

Observe that $g \in P$. Since $f(x) g(x)=f(x) \neq 0$ and for every point $t \neq x$ we have $f(t) g(t)=0$ or $|f(t) g(t)-f(x) g(x)|=|f(t)-f(x)|>\eta$, the function $f g$ is not s.q.c. at $x$, so $f g$ is not in $P$. This completes the proof. 
Remark 4. Let $f \in P$ be a function and let $x \in \mathcal{R}$ be a point such that $f(x)=0$. If $d_{u}(\{t ; f(t)=0\}, x)>0$ then for every function $g \in P$, for every positive real $\eta$ and for every set $A \in \mathcal{T}_{d}$ containing $x$ there is an open interval $I$ such that $I \cap A \neq \emptyset$, the product $f g \in \mathcal{P}(t)$ and $|f(t) g(t)|<\eta$ for each point $t \in I \cap A$.

Proof. Fix a function $g \in P$, a positive real $\eta$ and a set $A \in \mathcal{T}_{d}$ containing $x$. The functions $f, g \in P$, so they are almost everywhere continuous. Observe that the set $B=\{t ; t \in A, f(t)=0$ and $f$ is continuous at $t\}$ is of positive measure. There are a nonempty set $D \subset B$ belonging to $\mathcal{T}_{d}$ and a point $u \in D$ such that $f(u)=0$ and the function $g$ is continuous at $u$. Let $J$ be an open interval containing $u$ such that there is a positive real $K$ with $|g(t)|<K$ for all points $t \in J$. Evidently, $u \in J \cap A \in \mathcal{T}_{d}$. Since $f \in P$ and $f(u)=0$, there is an open interval $I_{1} \subset J$ such that $I_{1} \cap A \neq \emptyset, f \in \mathcal{P}(t)$, and $|f(t)|<\eta / K$ for all points $t \in I_{1} \cap A$. But $g \in P$ and $\emptyset \neq I_{1} \cap A \in \mathcal{T}_{d}$, so there is an open interval $I \subset I_{1}$ such that $I \cap A \neq \emptyset$ and $g \in \mathcal{P}(t)$ for each point $t \in I \cap A$. For $t \in I \cap A$ we have $f g \in \mathcal{P}(t)$ and $|f(t) g(t)-f(x) g(x)|=|f(t) g(t)|<(\eta / K) K=\eta$. This completes the proof.

In the proof next Remark 4 we will apply the following Lemma which is proved in [4] :

Lemma 1. Let $A \subset \mathcal{R}$ be a closed set and let $x \in A$ be a point such that $d_{u}(A, x)=0$. Then there is a sequence of disjoint closed intervals $I_{n}=$ $\left[a_{n}, b_{n}\right] \subset(x-2, x+2), n=1,2, \ldots$, such that:

$-\lim _{n \rightarrow \infty} a_{n}=\lim _{n \rightarrow \infty} b_{n}=x$;

$-d_{u}\left(\bigcup_{n} I_{n}, x\right)=0$

$-(A \backslash\{x\}) \cap[x-1, x+1] \subset \bigcup_{n} \operatorname{int}\left(I_{n}\right)$.

Remark 5. Suppose that a function $f \in P$ is not $\mathcal{T}_{a e}-$ continuous at a point $x$ at which $f(x)=0$. If

$$
d_{u}(\{t ; f(t)=0\}, x)=0
$$

then there is a function $g \in P$ such that the product $f g$ is not in $P$.

Proof. Since $f$ is almost everywhere continuous, we obtain

$$
\mu(c l(\{t ; f(t)=0\} \backslash\{t ; f(t)=0\})=0
$$

and

$$
d_{u}(c l(\{t ; f(t)=0\}), x)=0 .
$$

By Lemma 1 there are disjoint closed intervals $I_{n}=\left[a_{n}, b_{n}\right] \subset(x-2, x+$ 2) $\backslash\{x\}, n=1,2, \ldots$, such that 


$$
\begin{aligned}
& -\lim _{n \rightarrow \infty} a_{n}=\lim _{n \rightarrow \infty} b_{n}=x ; \\
& -[x-1, x+1] \cap \operatorname{cl}(\{t ; f(t)=0\}) \backslash\{x\} \subset \bigcup_{n} \operatorname{int}\left(I_{n}\right) ; \\
& -d_{u}\left(\bigcup_{n} I_{n}, x\right)=0 .
\end{aligned}
$$

Since the function $f$ is not $\mathcal{T}_{a e}-$ continuous at $x$, there are a positive real $\eta$ and disjoint closed intervals $J_{n}=\left[c_{n}, d_{n}\right] \subset(\{t ;|f(t)| \geq \eta / 2\} \cap(x-1, x+$ 1)) $\backslash \bigcup_{k} I_{k}$ such that $\lim _{n \rightarrow \infty} c_{n}=\lim _{n \rightarrow \infty} d_{n}=x$ and $d_{u}\left(\bigcup_{n} J_{n}, x\right)>0$. Moreover, we can assume that $f$ is continuous at all points $a_{n}, b_{n}, c_{n}, d_{n}, n=$ $1,2, \ldots$

\section{Put}

$$
g(t)=\left\{\begin{array}{ccl}
\eta & \text { if } & (t=x) \vee\left(t \in J_{n}, n \geq 1\right) \\
1 & \text { if } & (t \leq x-1) \vee(t \geq x+1) \vee\left(t \in I_{n}, n \geq 1\right) \\
1 / f(t) & \text { otherwise. }
\end{array}\right.
$$

By the methods used above we can show that the function $g \in P$. But the product $f g$ is not s.q.c. at $x$, since $f(x) g(x)=0, f(t) g(t)=1$ for $t \in$ $(x-2, x+2) \backslash \bigcup_{n}\left(I_{n} \cup J_{n}\right) \backslash\{x\},|f(t) g(t)| \geq \eta^{2} / 2$ for $t \in J_{n}, n \geq 1$ and $d_{u}\left(\bigcup_{n} I_{n}, x\right)=0$. So, the product $f g$ is not in $P$ and the proof is finished.

Remark 6. If a function $f \in P$ is $\mathcal{T}_{a e}-$ continuous at a point $x$ then for all functions $g \in P$, for every set $A \in \mathcal{T}_{d}$ containing $x$ and for every positive real $\eta$ there is an open interval $I$ such that $I \cap A \neq \emptyset, f g \in \mathcal{P}(t)$ and $\mid f(t) g(t)-$ $f(x) g(x) \mid<\eta$ for all points $t \in I \cap A$.

Proof. Fix a positive real $\eta$, and a set $A \in T_{d}$ such that $x \in A$. Since $f$ is $\mathcal{T}_{a e}$-continuous at $x$, so $x$ is a density point of the set

$$
B=\operatorname{int}(\{t ;|f(t)-f(x)|<(\eta / 2)(1 /|c|+1)\}),
$$

where $c=g(x)$. Consequently, $x$ is a density point of the set $B \cap A$. Since $f \in P$, there is an open interval $I \subset B$ such that $I \cap A \neq \emptyset$ and $f \in \mathcal{P}(t)$ for all points $t \in I \cap A$. Let $g \in P$ be any function. Since $g \in P$, there is an open interval $J \subset I$ such that $J \cap A \neq \emptyset,|g(t)-g(x)|<(\eta / 2)(1 /|f(t)|+1)$ and $g \in \mathcal{P}(t)$ for all $t \in J \cap A$. Consequently we obtain $f g \in \mathcal{P}(t)$ and

$$
\begin{gathered}
|f(t) g(t)-f(x) g(x)| \leq|f(t)||g(t)-g(x)|+|g(x)||f(t)-g(t)| \\
<|f(t)|(\eta / 2)(1 /|f(t)|+1)+|g(x)|(\eta / 2)(1 /|g(x)|+1)<\eta
\end{gathered}
$$

for all $t \in J \cap A$. So, $f g \in P$ and the proof is completed.

From Remarks $1-6$ it follows immediately: 
Theorem 4. A function $f \in \operatorname{Max}_{m u l t}(P)$ if and only if it is in $P$ and satisfies the following condition:

$(F)$ if $f$ is not $\mathcal{T}_{a e}-$ continuous at a point $x$ then $f(x)=0$ and $d_{u}(\{t ; f(t)=$ $0\}, x)>0$.

Remark 7. If the property $\mathcal{P}(x)$ denotes that $f(x) \in \mathcal{R}$ then all above results are true for $P=Q_{s}$ (see [4]).

\section{References}

[1] Bruckner A.M.; Differentiation of real functions, Lectures Notes in Math.659 (1978), Springer-Verlag.

[2] Grande Z.; On strong quasi-continuity of functions of two variables, Real Anal. Exch. 21 No.2 (1995-96), 236-243;

[3] Grande Z.; Measurability, quasicontinuity and cliquishness of functions of two variables, Real Anal. Exch. 20 No.2 (1994-95),744-752;

[4] Grande Z.; On the maximal families for the class of strongly quasicontinuous functions, Real Anal. Exch. 20 No.2 (1995-96),

[5] O'Malley R.J.; Approximately differentiable functions. The $r$ topology, Pacific J. Math. 72 (1977), 207-222. 